\title{
ON THE STARK BROADENING OF K III AND Ca IV SPECTRAL LINES
}

\author{
MILAN S. DIMITRIJEVIĆ ${ }^{1,2, \star}$ \\ ${ }^{1}$ Astronomical Observatory, Belgrade, Serbia \\ ${ }^{2}$ Sorbonne Université, Observatoire de Paris, Université PSL, CNRS, LERMA, F-92190, Meudon, France
}

\begin{abstract}
Stark full widths at half maximum (FWHM) for ten multiplets of doubly charged potassium ion (K III), and 35 multiplets of triply charged calcium ion (Ca IV) have been calculated for electron density of $10^{17} \mathrm{~cm}^{-3}$ by using the symplified modified semiempirical (SMSE) method. The calculations were performed for temperatures from $5000 \mathrm{~K}$ to $80000 \mathrm{~K}$, for K III and for temperatures from $10000 \mathrm{~K}$ to $160000 \mathrm{~K}$ for Ca IV, for broadening by collisions with electrons. The results obtained here will be implemented in the STARK-B database which is a memeber of Virtual atomic and molecular data center (VAMDC).
\end{abstract}

Keywords: Stark broadening, Spectral lines, Line profiles, K III, Ca IV.

\section{INTRODUCTION}

Data on Stark broadening, or broadening of spectral lines by collisions with charged particles due to Stark effect in a fluctuating electric microfield are needed in many research fields in astrophysics, for laboratory plasma diagnostics, for design of lasers and investigations of laser produced plasma, in inertial fusion research as well as for different technologies which use plasmas, like light industry, and cutting, welding and melting metals using lasers.

Line broadening data for as much as possible larger number of spectral lines is needed because we do not know a priori the chemical composition of a stellar atmosphere. Stark broadening research of the lines of trace elements is also needed because with the development of space astronomy, instruments like Goddhard High Resolution Spectrograph (GHRS) on Hubble Space Telescope, produce spectral line profiles with unpreceded resolution and accuracy and with modern computers all calculations become more and more easier.

$\mathrm{K}$ III and Ca IV lnes are the two successive members of the Chlorine isoelectronic sequence. Fritzsche et al. (2006) underline the importance of atomic data for them in fusion research as well as for estimating the energy loss through impurity ions. Stark broadening data for their spectral lines are needed for laboratory plasma diagnostics, in astophysics, for fusion plasma research and in plasma physics.

Since there is no neither experimental nor theoetical data for Stark broadening of K III and Ca IV spectral lines, here are calculated Stark full widths at half maximum (FWHM) for 10 multiplets of K III, and 35 of Ca IV by using the simplified modified semiempirical method (Dimitrijević \& Konjević, 1987), since a sufficiently complete set of atomic data, needed for a more sophysticated calculation does not exist.
The obtained results will be included in the STARK-B (Sahal-Bréchot et al., 2015, 2018), a database for Stark widths and shifts of spectral lines broadened by collisions with charged particles.

\section{SYMPLIFIED MSE FORMULA}

When there is no data for more sophysticated theoretical methods, or when data for a lot of spectral lines are needed, what is the case especially in astrophysics e.g. for model atmosphere or radiative transfer calculations, might be very useful the simplified modified semiempirical formula (Dimitrijević \& Konjević, 1987) for Stark widths of isolated, singly, and multiply charged ion lines. This formula may be used in the case when the nearest atomic energy level $\left(j^{\prime}=i^{\prime}\right.$ or $\left.f^{\prime}\right)$, having an allowed dipol transition from or to the initial $(i)$ or final $(f)$ energy level of the considered spectral line, is far enough, so that the condition $x_{\mathrm{jj},}=E /\left|E_{\mathrm{j},}-E_{\mathrm{j}}\right| \leq 2$ is satisfied. Than, full width at half maximum is given as (Dimitrijević \& Konjević, 1987):

$$
\begin{aligned}
& W(\AA)=2 . 151 \times 10^{-8} \frac{\lambda^{2}(\mathrm{~cm}) N\left(\mathrm{~cm}^{-3}\right)}{T^{1 / 2}(\mathrm{~K})}\left(0.9-\frac{1.1}{Z}\right) \times \\
& \times \sum_{\mathrm{j}=\mathrm{i}, \mathrm{f}}\left(\frac{3 n_{\mathrm{j}}^{*}}{2 Z}\right)^{2}\left(n_{\mathrm{j}}^{* 2}-\ell_{\mathrm{j}}^{2}-\ell-1\right) .
\end{aligned}
$$

In the upper equation, $N$ and $T$ are the electron density and temperature, $E=3 k T / 2$ is the energy of perturbing electron, $Z-1$ is the ionic charge, $n$ the effective principal quantum number and $\lambda$ is the transition wavelength.

\section{RESULTS AND DISCUSSION}

By using the symplified modified semiempirical (SMSE) method (Dimitrijević \& Konjević, 1987), Stark full widths at half

\footnotetext{
^ Corresponding author: mdimitrijevic@aob.rs
} 
Table 1. This table gives electron-impact broadening (Stark broadening) Full Widths at Half Intensity Maximum (W) for K III spectral lines, for a perturber density of $10^{17} \mathrm{~cm}^{-3}$ and temperatures from 5000 to $80000 \mathrm{~K}$. The configuration is $3 \mathrm{~s}^{2} 3 \mathrm{p}^{4}\left({ }^{3} \mathrm{P}\right) \mathrm{n} \ell$. Also is given quantity $3 k T / 2 \Delta E$ for $T=20000 \mathrm{~K}$, where $\Delta E$ is the energy difference between closest perturbing level and the closer of initial and final levels.

\begin{tabular}{|c|c|c|c|}
\hline Transition & $\mathrm{T}(\mathrm{K})$ & $\mathrm{W}[\AA]$ & $3 \mathrm{kT} / 2 \Delta \mathrm{E}$ \\
\hline $\mathrm{K}$ III $3 \mathrm{~d}^{2} \mathrm{~F}-4 \mathrm{p}^{2} \mathrm{D}^{o}$ & 5000. & $0.732 \mathrm{E}-01$ & 2.98 \\
\hline \multirow[t]{4}{*}{$\lambda=2366.6 \AA$} & 10000. & $0.518 \mathrm{E}-01$ & 5.95 \\
\hline & 20000. & $0.366 \mathrm{E}-01$ & 11.9 \\
\hline & 40000. & $0.259 \mathrm{E}-01$ & 23.8 \\
\hline & 80000. & $0.183 \mathrm{E}-01$ & 47.6 \\
\hline $\mathrm{K}$ III $4 \mathrm{~s}^{2} \mathrm{P}-4 \mathrm{p}^{2} \mathrm{D}^{o}$ & 5000. & 0.328 & 2.98 \\
\hline \multirow[t]{4}{*}{$\lambda=3312.2 \AA$} & 10000. & 0.232 & 5.95 \\
\hline & 20000. & 0.164 & 11.9 \\
\hline & 40000. & 0.116 & 23.8 \\
\hline & 80000. & $0.820 \mathrm{E}-01$ & 47.6 \\
\hline \multirow{5}{*}{$\begin{array}{c}\mathrm{K} \text { III } 4 \mathrm{~s}^{2} \mathrm{P}-4 \mathrm{p}^{2} \mathrm{P}^{o} \\
\lambda=3205.3 \AA\end{array}$} & 5000. & 0.311 & 1.89 \\
\hline & 10000. & 0.220 & 3.78 \\
\hline & 20000. & 0.155 & 7.56 \\
\hline & 40000 . & 0.110 & 15.1 \\
\hline & 80000. & $0.777 \mathrm{E}-01$ & 30.2 \\
\hline \multirow{5}{*}{$\begin{array}{c}\mathrm{K} \text { III } 4 \mathrm{p}^{2} \mathrm{D}^{o}-5 \mathrm{~s}^{2} \mathrm{P} \\
\lambda=5070.3 \AA\end{array}$} & 5000. & 1.23 & 2.98 \\
\hline & 10000. & 0.873 & 5.95 \\
\hline & 20000. & 0.617 & 11.9 \\
\hline & 40000. & 0.436 & 23.8 \\
\hline & 80000. & 0.309 & 47.6 \\
\hline \multirow{5}{*}{$\begin{array}{c}\mathrm{K} \text { III } 4 \mathrm{p}^{2} \mathrm{P}^{o}-5 \mathrm{~s}^{2} \mathrm{P} \\
\lambda=5342.9 \AA\end{array}$} & 5000. & 1.38 & 1.89 \\
\hline & 10000. & 0.976 & 3.78 \\
\hline & 20000. & 0.690 & 7.56 \\
\hline & 40000 . & 0.488 & 15.1 \\
\hline & 80000. & 0.345 & 30.2 \\
\hline \multirow{5}{*}{$\begin{array}{c}\mathrm{K} \text { III } 4 \mathrm{p}^{2} \mathrm{D}^{o}-3 \mathrm{~d}^{2} \mathrm{D} \\
\lambda=1678.3 \AA\end{array}$} & 5000. & 0.182 & 2.98 \\
\hline & 10000. & 0.129 & 5.95 \\
\hline & 20000. & $0.911 \mathrm{E}-01$ & 11.9 \\
\hline & 40000 . & $0.644 \mathrm{E}-01$ & 23.8 \\
\hline & 80000. & $0.456 \mathrm{E}-01$ & 47.6 \\
\hline \multirow{5}{*}{$\begin{array}{c}\text { K III } 4 \mathrm{p}^{2} \mathrm{P}^{o}-3 \mathrm{~d}^{2} \mathrm{D} \\
\lambda=1707.1 \AA\end{array}$} & 5000. & 0.190 & 1.89 \\
\hline & 10000. & 0.134 & 3.78 \\
\hline & 20000 . & $0.948 \mathrm{E}-01$ & 7.56 \\
\hline & 40000 . & $0.670 \mathrm{E}-01$ & 15.1 \\
\hline & 80000. & $0.474 \mathrm{E}-01$ & 30.2 \\
\hline \multirow{5}{*}{$\begin{array}{c}\mathrm{K} \text { III } 4 \mathrm{~s}^{4} \mathrm{P}-4 \mathrm{p}^{4} \mathrm{P}^{o} \\
\lambda=3376.2 \AA\end{array}$} & 5000. & 0.310 & 0.176 \\
\hline & 10000 . & 0.219 & 0.352 \\
\hline & 20000 . & 0.155 & 0.704 \\
\hline & 40000 . & 0.110 & 1.41 \\
\hline & 80000 . & $0.776 \mathrm{E}-01$ & 2.82 \\
\hline \multirow{5}{*}{$\begin{array}{c}\mathrm{K} \text { III } 4 \mathrm{~s}^{4} \mathrm{P}-4 \mathrm{p}^{4} \mathrm{D}^{o} \\
\lambda=3006.0 \AA\end{array}$} & 5000. & 0.256 & 0.176 \\
\hline & 10000 . & 0.181 & 0.352 \\
\hline & 20000 . & 0.128 & 0.704 \\
\hline & 40000 . & $0.905 \mathrm{E}-01$ & 1.41 \\
\hline & 80000 . & $0.640 \mathrm{E}-01$ & 2.82 \\
\hline \multirow{5}{*}{$\begin{array}{c}\text { K III } 4 \mathrm{~s}^{4} \mathrm{P}-4 \mathrm{p}^{4} \mathrm{~S}^{o} \\
\lambda=2601.3 \AA\end{array}$} & 5000. & 0.203 & 0.176 \\
\hline & 10000. & 0.144 & 0.352 \\
\hline & 20000 . & 0.102 & 0.704 \\
\hline & 40000 . & $0.719 \mathrm{E}-01$ & 1.41 \\
\hline & 80000. & $0.508 \mathrm{E}-01$ & 2.82 \\
\hline
\end{tabular}


Table 2. This table gives electron-impact broadening (Stark broadening) Full Widths at Half Intensity Maximum (W) for Ca IV spectral lines, for a perturber density of $10^{17} \mathrm{~cm}^{-3}$ and temperatures from 10000 to $160000 \mathrm{~K}$. Also is given quantity $3 k T / 2 \Delta E$, where $\Delta E$ is the energy difference between closest perturbing level and the closer of initial and final levels.

\begin{tabular}{|c|c|c|c|}
\hline Transition & $\mathrm{T}(\mathrm{K})$ & $\mathrm{W}[\AA]$ & $3 \mathrm{kT} / 2 \Delta \mathrm{E}$ \\
\hline Ca IV $\left({ }^{3} \mathrm{P}\right) 3 \mathrm{~d}^{4} \mathrm{D}-\left({ }^{3} \mathrm{P}\right) 4 \mathrm{p}^{4} \mathrm{P}^{o}$ & 10000. & $0.401 \mathrm{E}-02$ & 0.270 \\
\hline \multirow[t]{4}{*}{$\lambda=773.3 \AA$} & 20000. & $0.284 \mathrm{E}-02$ & 0.539 \\
\hline & 40000. & $0.201 \mathrm{E}-02$ & 1.08 \\
\hline & 80000. & $0.142 \mathrm{E}-02$ & 2.16 \\
\hline & 160000. & $0.100 \mathrm{E}-02$ & 4.31 \\
\hline Ca IV $\left({ }^{3} \mathrm{P}\right) 3 \mathrm{~d}^{4} \mathrm{D}-\left({ }^{3} \mathrm{P}\right) 4 \mathrm{p}^{4} \mathrm{D}^{o}$ & 10000. & $0.402 \mathrm{E}-02$ & 0.239 \\
\hline \multirow[t]{4}{*}{$\lambda=745.2 \AA$} & 20000. & $0.284 \mathrm{E}-02$ & 0.479 \\
\hline & 40000. & $0.201 \mathrm{E}-02$ & 0.958 \\
\hline & 80000. & $0.142 \mathrm{E}-02$ & 1.92 \\
\hline & 160000. & $0.100 \mathrm{E}-02$ & 3.83 \\
\hline \multirow{5}{*}{$\begin{array}{c}\mathrm{Ca} \text { IV }\left({ }^{3} \mathrm{P}\right) 3 \mathrm{~d}^{4} \mathrm{~F}-\left({ }^{3} \mathrm{P}\right) 4 \mathrm{p}^{4} \mathrm{D}^{o} \\
\lambda=858.2 \AA\end{array}$} & 10000. & $0.548 \mathrm{E}-02$ & 0.239 \\
\hline & 20000. & $0.387 \mathrm{E}-02$ & 0.479 \\
\hline & 40000. & $0.274 \mathrm{E}-02$ & 0.958 \\
\hline & 80000. & $0.194 \mathrm{E}-02$ & 1.92 \\
\hline & 160000. & $0.137 \mathrm{E}-02$ & 3.83 \\
\hline \multirow{5}{*}{$\begin{array}{c}\text { Ca IV }\left({ }^{3} \mathrm{P}\right) 3 \mathrm{~d}^{4} \mathrm{P}-\left({ }^{3} \mathrm{P}\right) 4 \mathrm{p}^{4} \mathrm{P}^{o} \\
\lambda=971.7 \AA\end{array}$} & 10000. & $0.663 \mathrm{E}-02$ & 0.270 \\
\hline & 20000. & $0.469 \mathrm{E}-02$ & 0.539 \\
\hline & 40000 . & $0.332 \mathrm{E}-02$ & 1.08 \\
\hline & 80000 . & $0.235 \mathrm{E}-02$ & 2.16 \\
\hline & 160000. & $0.166 \mathrm{E}-02$ & 4.31 \\
\hline \multirow{5}{*}{$\begin{array}{c}\text { Ca IV }\left({ }^{3} \mathrm{P}\right) 3 \mathrm{~d}^{4} \mathrm{P}-\left({ }^{3} \mathrm{P}\right) 4 \mathrm{p}^{4} \mathrm{D}^{o} \\
\lambda=927.7 \AA\end{array}$} & 10000. & $0.650 \mathrm{E}-02$ & 0.239 \\
\hline & 20000. & $0.460 \mathrm{E}-02$ & 0.479 \\
\hline & 40000 . & $0.325 \mathrm{E}-02$ & 0.958 \\
\hline & 80000. & $0.230 \mathrm{E}-02$ & 1.92 \\
\hline & 160000. & $0.163 \mathrm{E}-02$ & 3.83 \\
\hline \multirow{5}{*}{$\begin{array}{c}\text { Ca IV }\left({ }^{3} \mathrm{P}\right) 3 \mathrm{~d}^{4} \mathrm{P}-\left({ }^{3} \mathrm{P}\right) 4 \mathrm{p}^{4} \mathrm{~S}^{o} \\
\lambda=871.4 \AA\end{array}$} & 10000. & $0.636 \mathrm{E}-02$ & 0.206 \\
\hline & 20000. & $0.450 \mathrm{E}-02$ & 0.413 \\
\hline & 40000 . & $0.318 \mathrm{E}-02$ & 0.826 \\
\hline & 80000. & $0.225 \mathrm{E}-02$ & 1.65 \\
\hline & 160000 & $0.159 \mathrm{E}-02$ & 3.30 \\
\hline \multirow{5}{*}{$\begin{array}{c}\mathrm{Ca} \text { IV }\left({ }^{3} \mathrm{P}\right) 4 \mathrm{~s}^{4} \mathrm{P}-\left({ }^{3} \mathrm{P}\right) 4 \mathrm{p}^{4} \mathrm{P}^{o} \\
\lambda=2586.1 \AA\end{array}$} & 10000 . & 0.112 & 0.270 \\
\hline & 20000. & $0.794 \mathrm{E}-01$ & 0.539 \\
\hline & 40000 . & $0.561 \mathrm{E}-01$ & 1.08 \\
\hline & 80000 . & $0.397 \mathrm{E}-01$ & 2.16 \\
\hline & 160000 & $0.281 \mathrm{E}-01$ & 4.31 \\
\hline \multirow{5}{*}{$\begin{array}{c}\mathrm{Ca} \text { IV }\left({ }^{3} \mathrm{P}\right) 4 \mathrm{~s}^{4} \mathrm{P}-\left({ }^{3} \mathrm{P}\right) 4 \mathrm{p}^{4} \mathrm{D}^{o} \\
\lambda=2295.8 \AA\end{array}$} & 10000. & $0.913 \mathrm{E}-01$ & 0.270 \\
\hline & 20000. & $0.645 \mathrm{E}-01$ & 0.539 \\
\hline & 40000 . & $0.456 \mathrm{E}-01$ & 1.08 \\
\hline & 80000 . & $0.323 \mathrm{E}-01$ & 2.16 \\
\hline & 160000 & $0.228 \mathrm{E}-01$ & 4.31 \\
\hline \multirow{5}{*}{$\begin{array}{c}\text { Ca IV }\left({ }^{3} \mathrm{P}\right) 4 \mathrm{~s}^{4} \mathrm{P}-\left({ }^{3} \mathrm{P}\right) 4 \mathrm{p}^{4} \mathrm{~S}^{o} \\
\lambda=1979.5 \AA\end{array}$} & 10000. & $0.711 \mathrm{E}-01$ & 0.270 \\
\hline & 20000 . & $0.503 \mathrm{E}-01$ & 0.539 \\
\hline & 40000 . & $0.355 \mathrm{E}-01$ & 1.08 \\
\hline & 80000 . & $0.251 \mathrm{E}-01$ & 2.16 \\
\hline & 160000. & $0.178 \mathrm{E}-01$ & 4.31 \\
\hline \multirow{5}{*}{$\begin{array}{c}\mathrm{Ca} \text { IV }\left({ }^{3} \mathrm{P}\right) 3 \mathrm{~d}^{2} \mathrm{~F}-\left({ }^{3} \mathrm{P}\right) 4 \mathrm{p}^{2} \mathrm{D}^{o} \\
\lambda=937.1 \AA\end{array}$} & 10000 . & $0.701 \mathrm{E}-02$ & 0.284 \\
\hline & 20000 . & $0.495 \mathrm{E}-02$ & 0.567 \\
\hline & 40000 . & $0.350 \mathrm{E}-02$ & 1.13 \\
\hline & 80000. & $0.248 \mathrm{E}-02$ & 2.27 \\
\hline & 160000 & $0.175 \mathrm{E}-02$ & 4.54 \\
\hline
\end{tabular}




\begin{tabular}{|c|c|c|c|}
\hline Transition & $\mathrm{T}(\mathrm{K})$ & $\mathrm{W}[\AA]$ & $3 \mathrm{kT} / 2 \Delta \mathrm{E}$ \\
\hline \multirow{5}{*}{$\begin{array}{c}\text { Ca IV }\left({ }^{3} \mathrm{P}\right) 3 \mathrm{~d}^{2} \mathrm{P}-\left({ }^{3} \mathrm{P}\right) 4 \mathrm{p}^{2} \mathrm{D}^{o} \\
\lambda=2231.7 \AA\end{array}$} & 10000. & $0.476 \mathrm{E}-01$ & 0.284 \\
\hline & 20000. & $0.336 \mathrm{E}-01$ & 0.567 \\
\hline & 40000. & $0.238 \mathrm{E}-01$ & 1.13 \\
\hline & 80000 . & $0.168 \mathrm{E}-01$ & 2.27 \\
\hline & 160000. & $0.119 \mathrm{E}-01$ & 4.54 \\
\hline \multirow{5}{*}{$\begin{array}{c}\mathrm{Ca} \operatorname{IV}\left({ }^{3} \mathrm{P}\right) 3 \mathrm{~d}^{2} \mathrm{P}-\left({ }^{3} \mathrm{P}\right) 4 \mathrm{p}^{2} \mathrm{P}^{o} \\
\lambda=2264.9 \AA\end{array}$} & 10000. & $0.486 \mathrm{E}-01$ & 0.289 \\
\hline & 20000. & $0.344 \mathrm{E}-01$ & 0.578 \\
\hline & 40000. & $0.243 \mathrm{E}-01$ & 1.16 \\
\hline & 80000 . & $0.172 \mathrm{E}-01$ & 2.31 \\
\hline & 160000. & $0.122 \mathrm{E}-01$ & 4.62 \\
\hline \multirow{5}{*}{$\begin{array}{c}\mathrm{Ca} \text { IV }\left({ }^{3} \mathrm{P}\right) 3 \mathrm{~d}^{2} \mathrm{P}-\left({ }^{3} \mathrm{P}\right) 4 \mathrm{p}^{2} \mathrm{~S}^{o} \\
\lambda=2071.5 \AA\end{array}$} & 10000. & $0.428 \mathrm{E}-01$ & 0.259 \\
\hline & 20000. & $0.303 \mathrm{E}-01$ & 0.518 \\
\hline & 40000. & $0.214 \mathrm{E}-01$ & 1.04 \\
\hline & 80000 . & $0.151 \mathrm{E}-01$ & 2.07 \\
\hline & 160000. & $0.107 \mathrm{E}-01$ & 4.15 \\
\hline \multirow{5}{*}{$\begin{array}{c}\mathrm{Ca} \text { IV }\left({ }^{3} \mathrm{P}\right) 3 \mathrm{~d}^{2} \mathrm{P}-\left({ }^{3} \mathrm{P}\right) 4 \mathrm{p}^{2} \mathrm{D}^{o} \\
\lambda=2715.7 \AA\end{array}$} & 10000. & $0.729 \mathrm{E}-01$ & 0.284 \\
\hline & 20000. & $0.516 \mathrm{E}-01$ & 0.567 \\
\hline & 40000. & $0.365 \mathrm{E}-01$ & 1.13 \\
\hline & 80000 . & $0.258 \mathrm{E}-01$ & 2.27 \\
\hline & 160000. & $0.182 \mathrm{E}-01$ & 4.54 \\
\hline \multirow{5}{*}{$\begin{array}{c}\mathrm{Ca} \text { IV }\left({ }^{3} \mathrm{P}\right) 3 \mathrm{~d}^{2} \mathrm{P}-\left({ }^{3} \mathrm{P}\right) 4 \mathrm{p}^{2} \mathrm{P}^{o} \\
\lambda=2765.1 \AA\end{array}$} & 10000. & $0.750 \mathrm{E}-01$ & 0.289 \\
\hline & 20000. & $0.531 \mathrm{E}-01$ & 0.578 \\
\hline & 40000. & $0.375 \mathrm{E}-01$ & 1.16 \\
\hline & 80000. & $0.265 \mathrm{E}-01$ & 2.31 \\
\hline & 160000. & $0.188 \mathrm{E}-01$ & 4.62 \\
\hline \multirow{5}{*}{$\begin{array}{c}\mathrm{Ca} \text { IV }\left({ }^{3} \mathrm{P}\right) 4 \mathrm{~s}^{2} \mathrm{P}-\left({ }^{3} \mathrm{P}\right) 4 \mathrm{p}^{2} \mathrm{D}^{o} \\
\lambda=2720.8 \AA\end{array}$} & 10000. & 0.136 & 0.284 \\
\hline & 20000 . & $0.965 \mathrm{E}-01$ & 0.567 \\
\hline & 40000 . & $0.682 \mathrm{E}-01$ & 1.13 \\
\hline & 80000 . & $0.482 \mathrm{E}-01$ & 2.27 \\
\hline & 160000. & $0.341 \mathrm{E}-01$ & 4.54 \\
\hline \multirow{5}{*}{$\begin{array}{c}\text { Ca IV }\left({ }^{3} \mathrm{P}\right) 4 \mathrm{~s}^{2} \mathrm{P}-\left({ }^{3} \mathrm{P}\right) 4 \mathrm{p}^{2} \mathrm{P}^{o} \\
\lambda=2770.3 \AA\end{array}$} & 10000. & 0.141 & 0.289 \\
\hline & 20000. & $0.996 \mathrm{E}-01$ & 0.578 \\
\hline & 40000. & $0.704 \mathrm{E}-01$ & 1.16 \\
\hline & 80000. & $0.498 \mathrm{E}-01$ & 2.31 \\
\hline & 160000. & $0.352 \mathrm{E}-01$ & 4.62 \\
\hline \multirow{5}{*}{$\begin{array}{c}\mathrm{Ca} \text { IV }\left({ }^{3} \mathrm{P}\right) 4 \mathrm{~s}^{2} \mathrm{P}-\left({ }^{3} \mathrm{P}\right) 4 \mathrm{p}^{2} \mathrm{~S}^{o} \\
\lambda=2486.3 \AA\end{array}$} & 10000. & 0.117 & 0.284 \\
\hline & 20000. & $0.824 \mathrm{E}-01$ & 0.567 \\
\hline & 40000. & $0.583 \mathrm{E}-01$ & 1.13 \\
\hline & 80000 . & $0.412 \mathrm{E}-01$ & 2.27 \\
\hline & 160000. & $0.291 \mathrm{E}-01$ & 4.54 \\
\hline \multirow{5}{*}{$\begin{array}{c}\mathrm{Ca} \text { IV }\left({ }^{1} \mathrm{D}\right) 3 \mathrm{~d}^{2} \mathrm{P}-\left({ }^{1} \mathrm{D}\right) 4 \mathrm{p}^{2} \mathrm{D}^{o} \\
\lambda=730.6 \AA\end{array}$} & 10000. & 0.729E-02 & 0.229 \\
\hline & 20000. & $0.516 \mathrm{E}-02$ & 0.457 \\
\hline & 40000. & $0.365 \mathrm{E}-02$ & 0.915 \\
\hline & 80000. & $0.258 \mathrm{E}-02$ & 1.83 \\
\hline & 160000. & $0.182 \mathrm{E}-02$ & 3.66 \\
\hline \multirow{5}{*}{$\begin{array}{c}\text { Ca IV }\left({ }^{1} \mathrm{D}\right) 3 \mathrm{~d}^{2} \mathrm{P}-\left({ }^{1} \mathrm{D}\right) 4 \mathrm{p}^{2} \mathrm{P}^{o} \\
\lambda=720.4 \AA\end{array}$} & 10000. & $0.721 \mathrm{E}-02$ & 0.219 \\
\hline & 20000 . & $0.510 \mathrm{E}-02$ & 0.439 \\
\hline & 40000. & $0.361 \mathrm{E}-02$ & 0.877 \\
\hline & 80000 . & $0.255 \mathrm{E}-02$ & 1.75 \\
\hline & 160000. & $0.180 \mathrm{E}-02$ & 3.51 \\
\hline
\end{tabular}




\begin{tabular}{|c|c|c|c|}
\hline Transition & $\mathrm{T}(\mathrm{K})$ & $\mathrm{W}[\AA]$ & $3 \mathrm{kT} / 2 \Delta \mathrm{E}$ \\
\hline \multirow{5}{*}{$\begin{array}{c}\mathrm{Ca} \text { IV }\left({ }^{1} \mathrm{D}\right) 3 \mathrm{~d}^{2} \mathrm{D}-\left({ }^{1} \mathrm{D}\right) 4 \mathrm{p}^{2} \mathrm{D}^{o} \\
\lambda=813.2 \AA\end{array}$} & 10000. & $0.876 \mathrm{E}-02$ & 0.262 \\
\hline & 20000. & $0.620 \mathrm{E}-02$ & 0.523 \\
\hline & 40000 . & $0.438 \mathrm{E}-02$ & 1.05 \\
\hline & 80000 . & $0.310 \mathrm{E}-02$ & 2.09 \\
\hline & 160000. & $0.219 \mathrm{E}-02$ & 4.19 \\
\hline \multirow{5}{*}{$\begin{array}{c}\text { Ca IV }\left({ }^{1} \mathrm{D}\right) 3 \mathrm{~d}^{2} \mathrm{P}-\left({ }^{1} \mathrm{D}\right) 4 \mathrm{p}^{2} \mathrm{D}^{o} \\
\lambda=776.9 \AA\end{array}$} & 10000. & $0.839 \mathrm{E}-02$ & 0.229 \\
\hline & 20000. & $0.593 \mathrm{E}-02$ & 0.457 \\
\hline & 40000. & $0.419 \mathrm{E}-02$ & 0.915 \\
\hline & 80000. & $0.297 \mathrm{E}-02$ & 1.83 \\
\hline & 160000. & $0.210 \mathrm{E}-03$ & 3.66 \\
\hline \multirow{5}{*}{$\begin{array}{c}\mathrm{Ca} \text { IV }\left({ }^{1} \mathrm{D}\right) 3 \mathrm{~d}^{2} \mathrm{P}-\left({ }^{1} \mathrm{D}\right) 4 \mathrm{p}^{2} \mathrm{P}^{o} \\
\lambda=765.3 \AA\end{array}$} & 10000. & $0.828 \mathrm{E}-02$ & 0.219 \\
\hline & 20000. & $0.585 \mathrm{E}-02$ & 0.439 \\
\hline & 40000. & $0.414 \mathrm{E}-02$ & 0.877 \\
\hline & 80000 . & $0.293 \mathrm{E}-02$ & 1.75 \\
\hline & 160000. & $0.207 \mathrm{E}-02$ & 3.51 \\
\hline \multirow{5}{*}{$\begin{array}{c}\text { Ca IV }\left({ }^{1} \mathrm{D}\right) 3 \mathrm{~d}^{2} \mathrm{G}-\left({ }^{1} \mathrm{D}\right) 4 \mathrm{p}^{2} \mathrm{~F}^{o} \\
\lambda=848.5 \AA\end{array}$} & 10000. & $0.965 \mathrm{E}-02$ & 0.262 \\
\hline & 20000. & $0.682 \mathrm{E}-02$ & 0.523 \\
\hline & 40000 . & $0.483 \mathrm{E}-02$ & 1.05 \\
\hline & 80000. & $0.341 \mathrm{E}-02$ & 2.09 \\
\hline & 160000. & $0.241 \mathrm{E}-02$ & 4.19 \\
\hline \multirow{5}{*}{$\begin{array}{c}\mathrm{Ca} \text { IV }\left({ }^{1} \mathrm{D}\right) 3 \mathrm{~d}^{2} \mathrm{~F}-\left({ }^{1} \mathrm{D}\right) 4 \mathrm{p}^{2} \mathrm{~F}^{o} \\
\lambda=1002.2 \AA\end{array}$} & 10000. & $0.141 \mathrm{E}-01$ & 0.262 \\
\hline & 20000. & $0.996 \mathrm{E}-02$ & 0.523 \\
\hline & 40000 . & $0.704 \mathrm{E}-02$ & 1.05 \\
\hline & 80000 . & $0.498 \mathrm{E}-02$ & 2.09 \\
\hline & 160000. & $0.352 \mathrm{E}-02$ & 4.19 \\
\hline \multirow{5}{*}{$\begin{array}{c}\mathrm{Ca} \text { IV }\left({ }^{1} \mathrm{D}\right) 3 \mathrm{~d}^{2} \mathrm{~F}-\left({ }^{1} \mathrm{D}\right) 4 \mathrm{p}^{2} \mathrm{D}^{o} \\
\lambda=947.7 \AA\end{array}$} & 10000. & $0.132 \mathrm{E}-01$ & 0.229 \\
\hline & 20000. & $0.932 \mathrm{E}-02$ & 0.457 \\
\hline & 40000 . & $0.659 \mathrm{E}-02$ & 0.915 \\
\hline & 80000. & $0.466 \mathrm{E}-02$ & 1.83 \\
\hline & 160000. & $0.329 \mathrm{E}-02$ & 3.66 \\
\hline \multirow{5}{*}{$\begin{array}{c}\text { Ca IV }\left({ }^{1} \mathrm{D}\right) 3 \mathrm{~d}^{2} \mathrm{~S}-\left({ }^{1} \mathrm{D}\right) 4 \mathrm{p}^{2} \mathrm{P}^{o} \\
\lambda=1490.4 \AA\end{array}$} & 10000. & $0.373 \mathrm{E}-01$ & 0.219 \\
\hline & 20000. & $0.263 \mathrm{E}-01$ & 0.439 \\
\hline & 40000 . & $0.186 \mathrm{E}-01$ & 0.877 \\
\hline & 80000. & $0.132 \mathrm{E}-01$ & 1.75 \\
\hline & 160000. & $0.931 \mathrm{E}-02$ & 3.51 \\
\hline \multirow{5}{*}{$\begin{array}{c}\text { Ca IV }\left({ }^{1} \mathrm{D}\right) 4 \mathrm{~s}^{2} \mathrm{D}-\left({ }^{1} \mathrm{D}\right) 4 \mathrm{p}^{2} \mathrm{~F}^{o} \\
\lambda=2509.5 \AA\end{array}$} & 10000. & 0.108 & 0.262 \\
\hline & 20000. & $0.764 \mathrm{E}-01$ & 0.523 \\
\hline & 40000. & $0.540 \mathrm{E}-01$ & 1.05 \\
\hline & 80000 . & $0.382 \mathrm{E}-01$ & 2.09 \\
\hline & 160000. & $0.270 \mathrm{E}-01$ & 4.19 \\
\hline \multirow{5}{*}{$\begin{array}{c}\text { Ca IV }\left({ }^{1} \mathrm{D}\right) 4 \mathrm{~s}^{2} \mathrm{D}-\left({ }^{1} \mathrm{D}\right) 4 \mathrm{p}^{2} \mathrm{D}^{o} \\
\lambda=2193.5 \AA\end{array}$} & 10000. & $0.856 \mathrm{E}-01$ & 0.262 \\
\hline & 20000. & $0.606 \mathrm{E}-01$ & 0.523 \\
\hline & 40000 . & $0.428 \mathrm{E}-01$ & 1.05 \\
\hline & 80000. & $0.303 \mathrm{E}-01$ & 2.09 \\
\hline & 160000. & $0.214 \mathrm{E}-01$ & 4.19 \\
\hline \multirow{5}{*}{$\begin{array}{c}\text { Ca IV }\left({ }^{1} \mathrm{D}\right) 4 \mathrm{~s}^{2} \mathrm{D}-\left({ }^{1} \mathrm{D}\right) 4 \mathrm{p}^{2} \mathrm{P}^{o} \\
\lambda=2103.6 \AA\end{array}$} & 10000. & $0.798 \mathrm{E}-01$ & 0.262 \\
\hline & 20000 . & $0.564 \mathrm{E}-01$ & 0.523 \\
\hline & 40000. & $0.399 \mathrm{E}-01$ & 1.05 \\
\hline & 80000 . & $0.282 \mathrm{E}-01$ & 2.09 \\
\hline & 160000. & $0.200 \mathrm{E}-01$ & 4.19 \\
\hline
\end{tabular}




\begin{tabular}{crrr}
\hline Transition & $\mathrm{T}(\mathrm{K})$ & $\mathrm{W}[\AA]$ & $3 \mathrm{kT} / 2 \Delta \mathrm{E}$ \\
\hline Ca IV $\left({ }^{1} \mathrm{D}\right) 4 \mathrm{p}^{2} \mathrm{~F}^{o}-\left({ }^{1} \mathrm{D}\right) 5 \mathrm{~s}^{2} \mathrm{D}$ & 10000. & 0.126 & 0.262 \\
$\lambda=2091.3 \AA$ & 20000. & $0.892 \mathrm{E}-01$ & 0.523 \\
& 40000. & $0.631 \mathrm{E}-01$ & 1.05 \\
& 80000. & $0.446 \mathrm{E}-01$ & 2.09 \\
& 160000. & $0.315 \mathrm{E}-01$ & 4.19 \\
\hline Ca IV $\left({ }^{1} \mathrm{D}\right) 4 \mathrm{p}^{2} \mathrm{D}^{o}-\left({ }^{1} \mathrm{D}\right) 5 \mathrm{~s}^{2} \mathrm{D}$ & 10000. & 0.167 & 0.260 \\
$\lambda=2376.7 \AA$ & 20000. & 0.118 & 0.520 \\
& 40000. & $0.836 \mathrm{E}-01$ & 1.04 \\
& 80000. & $0.591 \mathrm{E}-01$ & 2.08 \\
& 160000. & $0.418 \mathrm{E}-01$ & 4.16 \\
\hline Ca IV $\left({ }^{1} \mathrm{D}\right) 4 \mathrm{p}^{2} \mathrm{P}^{o}-\left({ }^{1} \mathrm{D}\right) 5 \mathrm{~s}^{2} \mathrm{D}$ & 10000. & 0.185 & 0.260 \\
$\lambda=2492.0 \AA$ & 20000. & 0.131 & 0.520 \\
& 40000. & $0.927 \mathrm{E}-01$ & 1.04 \\
& 80000. & $0.655 \mathrm{E}-01$ & 2.08 \\
& 160000. & $0.463 \mathrm{E}-01$ & 4.16 \\
\hline $\mathrm{Ca} \mathrm{IV}\left({ }^{1} \mathrm{~S}\right) 3 \mathrm{~d}^{2} \mathrm{D}-\left({ }^{1} \mathrm{~S}\right) 4 \mathrm{p}^{2} \mathrm{P}^{o}$ & 10000. & $0.628 \mathrm{E}-02$ & 0.244 \\
$\lambda=912.0 \AA$ & 20000. & $0.444 \mathrm{E}-02$ & 0.488 \\
& 40000. & $0.314 \mathrm{E}-02$ & 0.976 \\
& 80000. & $0.222 \mathrm{E}-02$ & 1.95 \\
& 160000. & $0.157 \mathrm{E}-02$ & 3.90 \\
\hline $\mathrm{Ca} \mathrm{IV}\left({ }^{1} \mathrm{~S}\right) 4 \mathrm{~s}^{2} \mathrm{~S}-\left({ }^{1} \mathrm{~S}\right) 4 \mathrm{p}^{2} \mathrm{P}^{o}$ & 10000. & $0.953 \mathrm{E}-01$ & 0.244 \\
$\lambda=2339.8 \AA$ & 20000. & $0.674 \mathrm{E}-01$ & 0.488 \\
& 40000. & $0.476 \mathrm{E}-01$ & 0.976 \\
& 80000. & $0.337 \mathrm{E}-01$ & 1.95 \\
& 160000. & $0.238 \mathrm{E}-01$ & 3.9 \\
\hline & & &
\end{tabular}

maximum (FWHM) for 10 multiplets of K III and 35 multiplets of Ca IV have been calculated for electron density of $10^{17} \mathrm{~cm}^{-3}$ and for temperatures from $5000 \mathrm{~K}$ to $80000 \mathrm{~K}$ in the case of $\mathrm{K}$ III while for Ca IV the chosen temperature range was from 10 $000 \mathrm{~K}$ to $160000 \mathrm{~K}$. Calculations were performed for broadening of spectral lines by collisions with electrons. Energy levels and ionization energies needed for the corresponding calculations have been taken from Sugar \& Corlis (1985); Sansonetti (2008); Kramida et al. (2018) in the case of K III and from Sugar \& Corlis (1985); Kramida et al. (2018) in the case of Ca IV. The data for energy levels are incomplete for any more sophysticated calculation but using them, SMSE method, who needs less atomic data, can be applied.

The results of our calculations of Stark widths for K III spectral lines are shown in Table 1 and for Ca IV in Table 2. The extrapolation to lower perturber densities is linear while for higher extrapolation is linear to densities where the influence of Debye screening is negligible or reasonably small. The wavelengths presented in Tables 1 and 2 are the calculated ones from the averaged energy levels for multiplet so that they differ from the observed ones. In the last column is shown the quantity $3 k T / 2 \Delta E$, which represents the ratio of the average energy of free electrons, $E=3 k T / 2$, and the energy difference between the initial or final and the closest perturbing level,and is taken the larger of such values for initialand final level.
Table 3. This table gives electron-impact broadening (Stark broadening) Full Widths at Half Intensity Maximum (W) for K III $4 \mathrm{~s}^{(2 S+1)} \mathrm{P}-4 \mathrm{p}^{2 S+1} \mathrm{~L}^{o}(\mathrm{~L}=\mathrm{S}, \mathrm{P}, \mathrm{D})$ supermultiplet, for a perturber density of $10^{17} \mathrm{~cm}^{-3}$ and temperature of $10000 \mathrm{~K}$, in $[\AA]$ and in $\left[10^{12} \mathrm{~s}^{-1}\right]$ units.

\begin{tabular}{|c|c|c|c|}
\hline Transition & $\mathrm{T}(\mathrm{K})$ & $\mathrm{W}[\AA]$ & $\mathrm{W}\left[10^{12} \mathrm{~s}^{-1}\right]$ \\
\hline $\begin{array}{c}\mathrm{K} \text { III } 4 \mathrm{~s}^{2} \mathrm{P}-4 \mathrm{p}^{2} \mathrm{D}^{o} \\
\lambda=3312.2 \AA\end{array}$ & 10000. & 0.232 & 0.398 \\
\hline $\begin{array}{c}\mathrm{K} \mathrm{III} 4 \mathrm{~s}^{2} \mathrm{P}-4 \mathrm{p}^{2} \mathrm{P}^{o} \\
\lambda=3205.3 \AA\end{array}$ & 10000. & 0.220 & 0.403 \\
\hline $\begin{array}{c}\mathrm{K} \mathrm{III} 4 \mathrm{~s}^{4} \mathrm{P}-4 \mathrm{p}^{4} \mathrm{P}^{o} \\
\lambda=3376.2 \AA\end{array}$ & 10000. & 0.219 & 0.362 \\
\hline 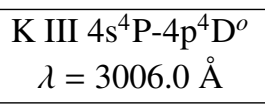 & 10000. & 0.181 & 0.377 \\
\hline $\begin{array}{c}\text { K III } 4 s^{4} \mathrm{P}-4 \mathrm{p}^{4} \mathrm{~S}^{o} \\
\lambda=2601.3 \AA\end{array}$ & 10000. & 0.144 & 0.401 \\
\hline
\end{tabular}

$$
\Delta E=\operatorname{Max}\left[E / \Delta E_{i, i^{\prime}}, E / \Delta E_{f, f^{\prime}}\right]
$$

This ratio is the validity condition for the used method. If $3 k T / 2 \Delta E=1$, it is the threshold for the corresponding inelastic transition. If it is lower than one, elastic collisions are dominant and it is so called low temperature limit, and the applied method is completely convenient and valid. If it is larger than one the inelas- 
tic collisions start to play more and more important role with its increase and the method underestimates the real value. However, since the Stark width decrease monotonically with the increase of temperature, if we look at the value of Stark width at lower temperatures, where the condition given by Eq. (2) is satisfied, and the obtained value for the needed temperature, the right value is between these limits, so that we have a rough estimate for it.

The Stark widths obtained here, enable to check the similarities of these line broadning parameters within a supermultiplet and transition array. If such similarities exist, they could be used for derrivation of missing values, on the basis of the known ones. Namely, Wiese \& Konjević (1982) found that Stark line widths in angular frequency units in a supermultiplet usually agree within about 30 per cent and within a transition array within about 40 per cent. We will check this for $\mathrm{K}$ III, belonging to the chlorine isoelectronic sequence, for the supermltiplet $4 \mathrm{~s}^{(2 S+1)} \mathrm{P}-4 \mathrm{p}^{2 S+1} \mathrm{~L}^{o}$ ( $\mathrm{L}=\mathrm{S}, \mathrm{P}, \mathrm{D})$. In order to do so we should transform the Stark widths expressed in $\AA$-units to the widths in angular frequency units, what can be done by the following formula:

$$
W(\AA)=\frac{\lambda^{2}}{2 \pi c} W\left(s^{-1}\right),
$$

where $c$ is the speed of light.

The values in $\AA$ and in $\mathrm{s}^{-1}$ are presented in Table 3. We can see that for values in $\AA$ the highest value in supermultiplet is $52 \%$ higher from the lowest and in transition array $61 \%$, but this is because of the influence of wavelength. In $\mathrm{s}^{-1}$ units, when the width is liberated from this influence, the maximal value in both supermultiplet and transition array is only $11 \%$ larger from the smaller one what is well within the limits found by Wiese \& Konjević (1982).

The Stark widths obtained here for K III and Ca IV spectral lines will be included in the STARK-B database (Sahal-Bréchot et al., 2015, 2018), one of the databases which enter also in the european Virtual Atomic and Molecular Data Center - VAMDC (Dubernet et al., 2010; Rixon et al., 2011; Dubernet et al., 2016), which can be found on the web site http://portal.vamdc.org/.

\section{CONCLUSION}

By using the SMSE method we have calculated Stark widths for $10 \mathrm{~K} \mathrm{III} \mathrm{and} 35 \mathrm{Ca}$ IV multiplets as a function of temperature. The presented widths are also used to check how similar they are within a supermultiplet and transition array. These data will be implemented in STARK-B database. Since other experimental or theoretical data for these ions do not exist it is not possible to make a comparison with other results. The data obtained in this work may be of interest for stellar physics, plasma diagnostics and inertial fusion reearch.

\section{REFERENCES}

Dimitrijević, M. S. \& Konjević, N. 1987. Simple estimates for Stark broadening of ion lines in stellar plasma. Astronomy and Astrophysics, 172, pp. 345-349.

Dubernet, M. L., Antony, B. K., Ba, Y. A., et al. 2016. The virtual atomic and molecular data centre (VAMDC) consortium. Journal of Physics B: Atomic, Molecular and Optical Physics, 49(7), 074003.

Dubernet, M. L., Boudon, V., Culhane, J. L., et al. 2010. Virtual atomic and molecular data centre. Journal of Quantitative Spectroscopy and Radiative Transfer, 111(15), pp. 2151-2159. doi:10.1016/j.jqsrt.2010.05.004.

Fritzsche, S., Finkbeiner, M., Fricke, B., \& Sepp, W. 2006. Level energies and lifetimes in the $3 \mathrm{p} 43 \mathrm{~d}$ configuration of chlorine-like ions. Physica Scripta, 52(3), pp. 258-266. doi:10.1088/0031-8949/52/3/006.

Kramida, A., Ralchenko, Y., \& Reader, J. 2018. NIST ASD Team, NIST Atomic Spectra Database. Gaithersburg, MD: National Institute of Standards and Technology. (ver. 5.5.1), Retrived from https://physics.nist.gov/asd, 2018, December 9.

Rixon, G., Dubernet, M. L., Piskunov, N., et al. 2011. VAMDCThe Virtual Atomic and Molecular Data Centre-A New Way to Disseminate Atomic and Molecular Data-VAMDC Level 1 Release. AIP Publishing., pp. 107-115. doi:10.1063/1.3585810.

Sahal-Bréchot, S., Dimitrijević, M. S., \& Moreau, N. 2018. STARK-B database. Observatory of Paris / LERMA.Astronomical Observatory of Belgrade. Retrieved from http://stark-b.obspm.fr, 2018 December 9.

Sahal-Bréchot, S., Dimitrijević, M. S., Moreau, N., \& Nessib, N. B. 2015. The STARK-B database VAMDC node: a repository for spectral line broadening and shifts due to collisions with charged particles. Physica Scripta, 90(5), p. 54008. doi:10.1088/0031-8949/90/5/054008.

Sansonetti, J. E. 2008. Wavelengths, Transition Probabilities, and Energy Levels for the Spectra of Potassium (K I through K XIX). J. Phys. Chem. Ref. Data, 37, pp. 7-96; DOI: 10.1063/1.2789451.

Sugar, J. \& Corlis, C. 1985. Atomic Energy Levels of the IronPeriod Elements: Potassium through Nickel. J . Phys. Chem. Ref. Data, 14, Suppl. 2, pp. 1-664.

Wiese, W. L. \& Konjević, N. 1982. Regularities and similarities in plasma broadened spectral line widths (Stark widths). Journal of Quantitative Spectroscopy and Radiative Transfer, 28(3), pp. 185-198. doi:10.1016/0022-4073(82)90022-x. 